\title{
Changes in bone density, intraosseous pressure of distal femoral articular cartilage and subchondral bone after proximal femoral medullary cavity cement filling in rabbits
}

\author{
GUO-PING LE ${ }^{1 *}$, LI-CHENG XI $^{1 *}$, MING ZHANG ${ }^{1}$ and JIN-MIN ZHAO ${ }^{2}$ \\ ${ }^{1}$ Department of Orthopedics, Fourth Affiliated Hospital of Guangxi Medical University, \\ Liuzhou, Guangxi 545005; ${ }^{2}$ Division of Traumatic Surgery, First Affiliated Hospital of \\ Guangxi Medical University, Nanning, Guangxi 530021, P.R. China
}

Received October 2, 2019; Accepted April 12, 2021

DOI: $10.3892 /$ etm.2021.10271

\begin{abstract}
Bone cement is widely used, particularly in hip replacements, but the potential clinical complications of its use have been largely unrecognized. The purpose of the present study was to investigate the effects of bone cement in the proximal femoral medullary cavity (PFMC) on bone mineral density (BMD), intraosseous pressure (IOP), articular cartilage and subchondral bone in the distal femurs of rabbits. A total of 32 New Zealand white rabbits were randomly numbered and the left hind limb of the odd-numbered rabbits and the right hind limb of the even numbered rabbits were selected as the experimental side. For each rabbit, the non-experimental hind limb was labeled as the control side by the principal investigator. An intramedullary injection of polymethyl methacrylate was made into the experimental hindlimb of each rabbit and the PFMC filled with bone cement. BMD and IOP of the distal femur of the bilateral hindlimb were measured at 4 and 16 weeks after surgery, and histological and ultra-fine structural features were examined by light and transmission electron microscopy, respectively. At week 4 after the operation, IOP in the experimental limb was significantly higher and BMD lower compared with the control limb. At the 16th week after operation, the IOP in the experimental limb was lower than at the 4th week after operation, but still higher compared with controls, and the BMD was significantly higher than the controls. In the controls, IOP and BMD was not significantly different between the 4th and 16th week after operation. Compared with controls, the cartilage in the experimental
\end{abstract}

Correspondence to: Professor Li-Cheng $\mathrm{Xi}$, Department of Orthopedics, Fourth Affiliated Hospital of Guangxi Medical University, 47 Hongyan Road, Liuzhou, Guangxi 545005, P.R. China E-mail: 469459442@qq.com

${ }^{*}$ Contributed equally

Key words: arthroplasty, bone cement, proximal femoral medullary cavity group was thinner, the chondrocytes partially necrotic and the trabecular structure of the subchondral bone broken. Analysis of ultra-fine structural features in the experimental group showed chondrocytes with necrotic cytoplasm and pyknotic nuclei relative to controls. The results indicated that blockage of the PFMC with bone cement resulted in an increase in the IOP in the distal femur, a change in BMD and damage to the subchondral bone and articular cartilage.

\section{Introduction}

Artificial hip arthroplasty is used extensively in replacement of damaged hips. To affix the artificial hip to the native bone, both cement and non-cement methods are used. Bone cement fixation is particularly suitable for aged patients with osteoporosis, as the early stability is generally good (1). With the development of new bone cement technology, the use of bone cement in artificial hip replacements is now more commonly accepted in clinics (2-4). However, bone cement in the proximal femoral medullary cavity (PFMC) can cause potential complications, such as high intraosseous pressure in the proximal femur and systemic bone cement implantation syndrome, including hypotension, arrhythmia, severe hypoxemia, myocardial infarction and increased pulmonary artery pressure (5-7). Previous studies have shown that a cement-fixed artificial joint prosthesis can lead to long-term occlusion of the medullary cavity and the destruction of intramedullary blood vessels (8). In addition, Yoon et al (9) have confirmed that the use of bone cement in total hip arthroplasty increases the risk of deep infection.

There are negative effects of bone cement on tissue around the cement at both the proximal and the distal end of the femur (10). In previous clinical observations, it was noted that some patients had pain and discomfort of the knee joint after artificial bone cement hip replacement (11), though the specific mechanisms underlying this were unclear. Our previous study found that blocking of PFMC with bone cement was associated with a significant decrease in blood circulation and bone metabolic rate of the distal femur (10). However, to the best of our knowledge, the effects of bone cement on bone mineral density (BMD), intraosseous pressure (IOP), articular 
cartilage and subchondral bone of the distal femur have not been explicitly reported. Therefore, in the present study, the effects of bone cement in the PFMC on the distal femur in rabbit models were assessed by measuring the BMD and IOP of the distal femur and observing changes in articular cartilage and subchondral bone structure using light microscopy (LM) and transmission electron microscopy (TEM).

\section{Materials and methods}

Ethical approval. The Institutional Animal Care and Use Committee approved the use of rabbits in this study, and the study protocol was approved by The Ethics Review Board of Guangxi Medical University (Nanning, China). The study complied with the National Institutes of Health Guide for Care and Use of Laboratory Animals (Publication No. 85-23) (12).

Animals and surgery. A total of 32 New Zealand white rabbits (weight, 2.6-3.5 kg; age, 6 months; 16 males and 16 females) were purchased from the Animal Center of Guangxi Medical University (Nanning, China). Rabbits were housed in individual cages at constant temperature $\left(18-22^{\circ} \mathrm{C}\right)$ and humidity (40-60\%) on a 12-h light-dark cycle with free access to food and water and were monitored every $24 \mathrm{~h}$.

The experimental rabbits were randomly numbered 1-32 and the left hind limb of the odd-numbered rabbits and the right hind limb of the even numbered rabbits was selected as the experimental side, whereas the other hind limb of the rabbit was labeled as the control side by the principal investigator; the surgeons and the independent researchers were both blinded to the identity of the rabbit.

Surgery was performed as described in a previous report (13): First, rabbits were anesthetized with an intramuscular injection of ketamine $(50 \mathrm{mg} / \mathrm{kg})$ and xylazine $(10 \mathrm{mg} / \mathrm{kg})$. The fur at the bilateral surgical site of the hind legs was then shaved after $9 \%$ sodium sulfide treatment, the operation field was further disinfected using iodophor and then covered with a surgical drape. The rabbits were placed on the surgical table on prone position. Using the third trochanter of the bilateral femur of the experimental rabbit as the central mark, a $4-\mathrm{cm}$ long arc incision was made. The lateral part of the trochanter was excised by a bone saw, and the entrance of the medullary cavity was established with a probe. The PFMC was created by repeatedly reaming with a medullary cavity file until the cavity reached about $3 / 5$ of the length of the femur. Polymethyl methacrylate (PMMA) solution was prepared by mixing two parts PMMA powder and one part PMMA liquid according to manufacturer's instructions (weight ratio, 2:1; DePuy International Ltd.). PMMA solution ( $2 \mathrm{ml}$ ) was injected into the medullary cavity of the right femur, which was then filled with the dough-like bone cement. The left femur was used as the control (without any bone cement). The wounds were washed with sterile saline, and the tissues were sutured to close the incision. After surgery, each rabbit received one injection of antibiotics, including $1 \mathrm{ml}$ gentamicin and 400,000 units of penicillin sodium. At the 4th or 16th week after operation, rabbits ( $n=16$ rabbits per time point) were anesthetized with an intramuscular injection of ketamine $(50 \mathrm{mg} / \mathrm{kg})$ and xylazine $(10 \mathrm{mg} / \mathrm{kg})$ and euthanized by air embolization by intravenously injecting $40-50 \mathrm{ml}$ of air into the auricle vein.
Death was confirmed by respiratory and cardiac arrest, pupil diffusion and disappearance of the nerve reflex.

IOP measurement. At the 4th or 16th week after operation, the rabbits were fixed on a custom-made operating table, and the skin and muscles were cut to expose the femoral medial condyle. At $1 \mathrm{~cm}$ above the femoral medial condyle, a bone puncture needle was inserted into the PFMC, and the needle was immediately connected to the pressure-measuring device tightly. Once the connection was established, the recording system was turned on and the pressure data recording was started (14). After the pressure signal was converted into an electrical signal, the pressure signal was input into a specially designed computer system to amplify and process, and the pressure data and curves could then be displayed (15). The IOP was recorded by a Physiological Pressure Transducer (SP844; MEMSCAP SA).

$H \& E$ staining and LM examination. The articular cartilage of the bilateral distal femur was harvested (including the subchondral bone). A $1 \mathrm{~cm}$ long cancellous bone mass of femoral condyle was selected near the articular surface. After rinsing the specimens with normal saline, the specimens were immediately fixed in $10 \%$ formaldehyde for $24-48 \mathrm{~h}$ at room temperature, followed by EDTA decalcification, washing with water and dehydration using a gradient of ethanol solutions (from 70-100\%). Following treatment with xylene I and xylene II for $30 \mathrm{~min}$ at room temperature the transparent tissues were paraffin embedded and sliced into $4-\mu \mathrm{m}$ thick sections using EM UC7 ultramicrotome (Leica Microsystems $\mathrm{GmbH})$. H\&E staining was carried out according to standard procedures (16). Briefly, slides were immersed in filtered Harris hematoxylin, rinsed with water, immersed in $0.3 \%$ ammonium hydroxide and rinsed with water. Subsequently, the sections were stained with eosin staining solution for 2-3 min, followed by 70 and $90 \%$ alcohol dehydration for $10 \mathrm{~min}$. Dehydrated slides were immersed in xylene and mounted using Permount ${ }^{\mathrm{TM}}$ mounting medium (Thermo Fisher Scientific, Inc.) and cover slips. Slides were observed under a light microscope (model, BX53; Olympus Corporation) after sealing.

TEM. The cancellous bone mass of the femoral condyle (including the articular cartilage and facial segment) near the articular surface was fixed for $2 \mathrm{~h}$ at room temperature with $2 \%$ glutaraldehyde (Panreac Química SLU) and 2.5\% paraformaldehyde at $\mathrm{pH} 7.4$ with $0.1 \mathrm{M}$ sodium cacodylate, rinsed with phosphate buffer and fixed in $1 \%$ osmium-acid solution. After rinsing, the bone mass was dehydrated using an ascending acetone series (50, 70, 90 and 100\%), soaked in a mixture of acetone/encapsulated solution, embedded in Epons12 embedding agent, and then sliced into 50-nm thick sections using an EM UC7 ultramicrotome (Leica Microsystems GmbH). The ultrastructure of cartilage and subchondral bone was observed using a JEM-1230 transmission electron microscope (JEOL Ltd.) according to standard procedures (17).

Bone mineral density (BMD) measurement. The cancellous bone mass of femoral condyle (including articular cartilage and facial subdivision) near the articular surface was selected for BMD measurement. Specifically, a $0.5 \mathrm{~cm}$ length piece 


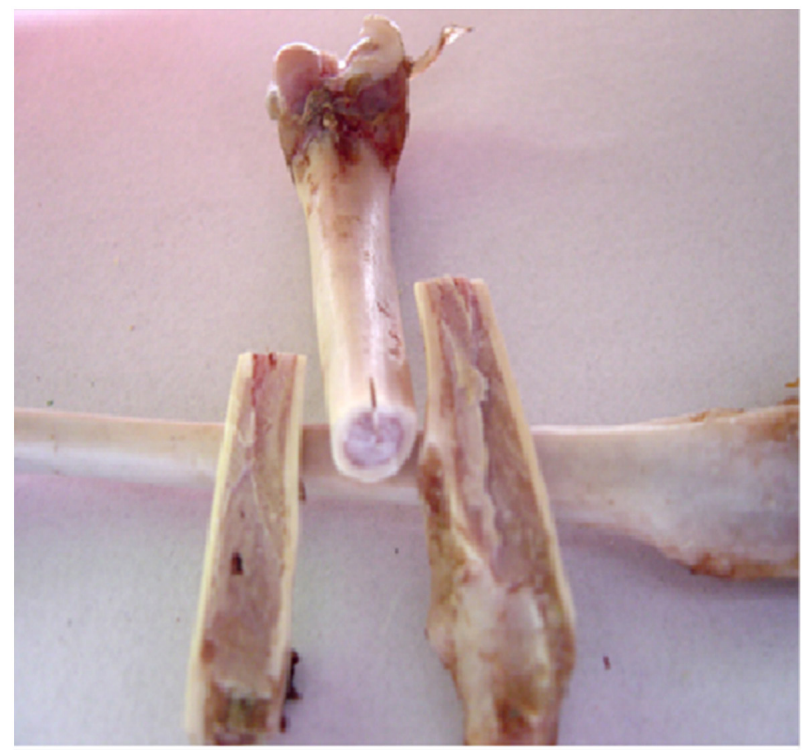

Figure 1. Gross observation of bone. The femur originated from the experimental side of the rabbit hind limb, and the anatomy of the gross specimen confirmed that the proximal femoral medullary cavity of the experimental side was filled with bone cement.

of intact cancellous bone of femoral condyle, $1 \mathrm{~cm}$ above the femoral condyle and close to the proximal articular surface, was used for BMD examination. The specimen was scanned using a high-resolution mode on a Hologic QDR 4500 fan-beam bone densitometer (DXA, Hologic, Inc.). The method was adapted from previous reports $(18,19)$.

Statistical analysis. All numerical data are presented as the mean \pm standard deviation. All statistical analyses were performed with SPSS18.0 statistical software (SPSS Inc.). Comparisons of BMD and IOP were analyzed by two-way ANOVA with Dunnett's post hoc test. $\mathrm{P}<0.05$ was considered to represent a statistically significant difference.

\section{Results}

Surgery. All animals tolerated the surgical procedure well. At 5 days post-operation, 2 rabbits had a minor infection at the incision site, but this healed well following treatment with antibiotics following debridement.

Gross anatomical morphology observation and X-ray of femurs in rabbits post-surgery. From the gross anatomical analysis of the femoral specimens, it was observed that the bone cement-filled bone marrow cavity was very tight without obvious gaps (Fig. 1). The presence of a bone cement shadow could be seen in X-ray images (Fig. 2); the upper middle femur of the experimental side of the rabbit was filled with cement, whereas the upper middle femur of the control side was not filled with cement, which demonstrated that the bone cement was filled to the middle segment of femur.

Evaluation of cartilage and bone tissue structural features by $L M$. At the 16th week post-surgery, it was found that the cartilage surface was smooth, chondrocytes were arranged in columnar shape and that the distribution of chondrocytes and the size of the nucleus was uniform on the control side (Fig. 4a). In control animals, the trabecular structure of the subchondral bone was normal, the bone cells were regularly arranged, the structure of bone was largely intact, and the lamellar line is clear (Fig. 3a). By contrast, chondrocytes in the experimental group were thinner and the surface layer was almost absent with a typical irregular moth-eaten shape (Fig. 4b). The arrangement of chondrocytes was disordered, the quantity was reduced, and a large number of chondrocytes were necrotic in the experimental group (Fig. 4b). A large number of empty bone lacunae in the subchondral trabeculae, a decrease in the number of bone cells, a disappearance of lamellar lines and broken trabecular structures were observed (Fig. 3b).

Evaluation of cartilage and bone tissue ultrastructural features by TEM. At the 16th week after intervention, the nuclei of some chondrocytes in the experimental group showed typical features of pyknosis and some cell and nuclear membranes could not be distinguished (Fig. 5b); overall, the general structure of the chondrocytes was disorganized, with necrotic and disintegrated cytoplasm (Fig. 5b). Similarly, the size of bone cells in the experimental group was reduced and the cell edges were unclear (Fig. 6b); the continuity of cell membrane and nuclear membranes was interrupted, with a disappearance of cytoplasmic components and the high-density substances in cells was increased (Fig. 6b). By contrast, the chondrocytes of the control group were more round-shaped, with an abundant rough endoplasmic reticulum and developed Golgi complex in the cytoplasm (Fig. 5a); the nucleus was large and complete and chromatin was abundant and evenly distributed throughout (Fig. 5a). Bone cells in the control group were located in the lacuna of bone with a flat oval shape, rich cytoplasm and endoplasmic reticulum, scattered free ribosomes, moderate mitochondria and well-developed Golgi complex (Fig. 6a).

Comparison of BMD of femurs between the two groups post-surgery. At the 4 th week following operation, the BMD of the distal femur in the experimental group $(0.275 \pm 0.005)$ was significantly lower compared with that in the control group (0.344 \pm 0.06$)(\mathrm{P}<0.05$; Fig. 7). However, at the 16th week post-operation, the BMD of the distal femur of the experimental group $(0.401 \pm 0.006)$ was significantly higher compared with the BMD at the 4 th week $(\mathrm{P}<0.05)$, and also higher than the control group at the 16th week $(0.355 \pm 0.005)$ $(\mathrm{P}<0.05)$. By contrast, there was no significant difference in BMD between the 4th and 16th week after operation in the control group ( $\mathrm{P}>0.05$; Fig. 7).

Comparison of IOP between the two groups post-surgery. At the 4th week post-operation, the IOP of the distal femur in the experimental group $(36.483 \pm 1.005)$ was significantly higher compared with that in the control group (21.972 \pm 0.640$)$ $(\mathrm{P}<0.05$; Fig. 8). However, at the 16th week following surgery, the IOP of the distal femur on the experimental side $(29.858 \pm 0.733)$ was lower compared with that at the 4 th week after the operation $(\mathrm{P}<0.05)$, but still higher compared with the control group at the 16 th week $(23.204 \pm 0.359)(\mathrm{P}<0.05)$. No 


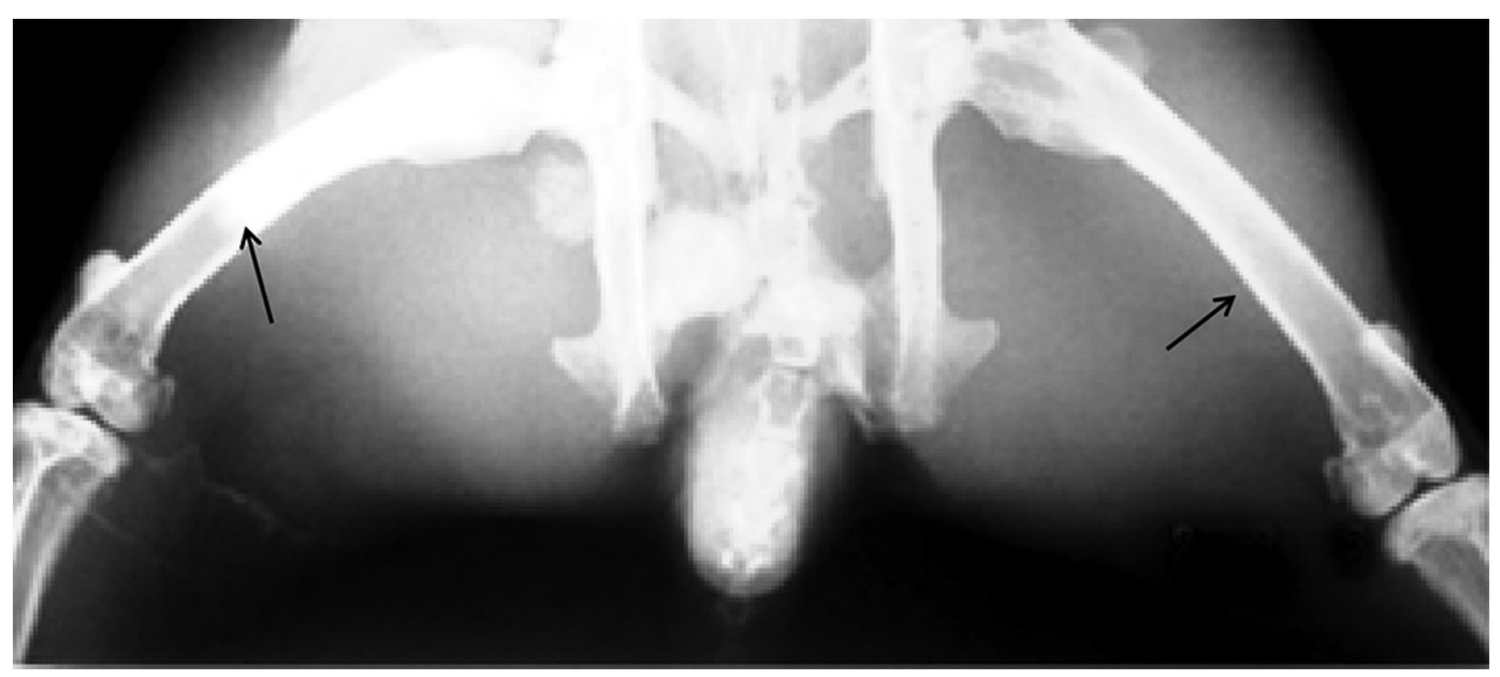

Figure 2. X-ray imaging of the femur. The X-ray imaging demonstrated that the proximal femoral medullary cavity of the experimental side was filled with bone cement (left), while the control side was not filled with bone cement (right). Arrows indicate the position of bone cement filling into the cavity.

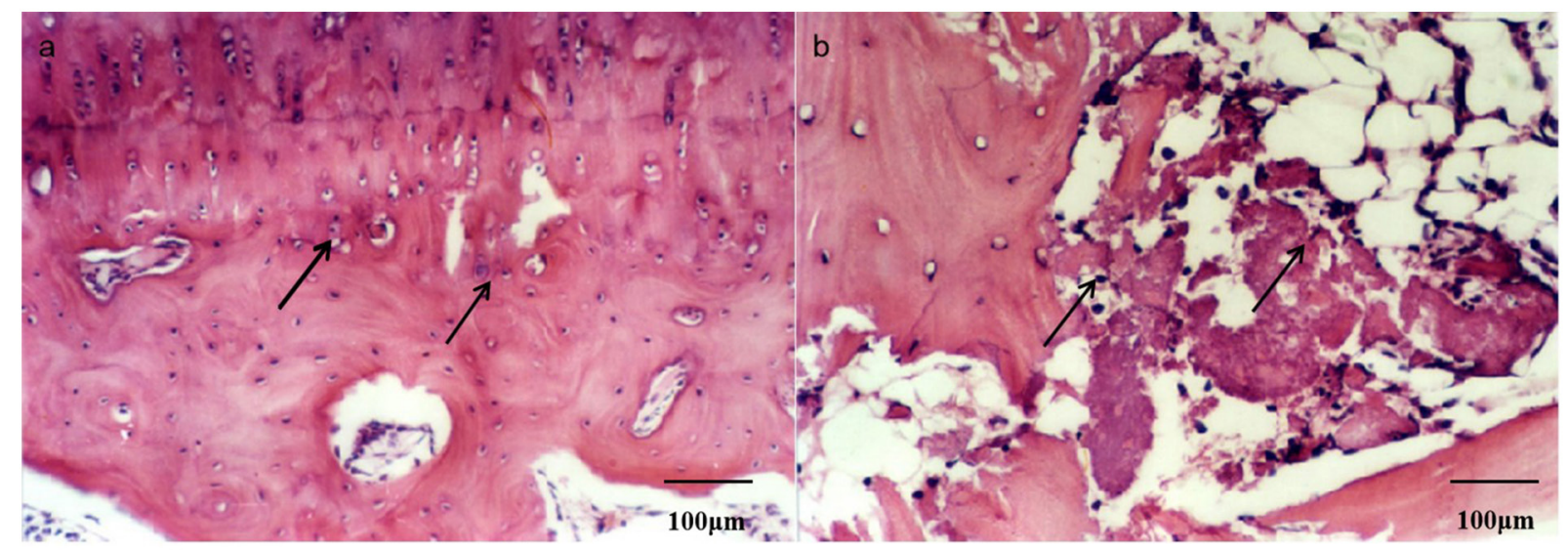

Figure 3. H\&E staining. (a) Subchondral bone structure of the control group under LM at the 16th week after intervention. The arrows indicate that the bone cells were well arranged, and the trabeculae were well structured. (b) Structure of articular subchondral bone in experimental group under LM at the 16th week after intervention. The arrows indicate a large number of empty bone lacunae in the subchondral trabeculae, the number of osteocytes being decreased compared with the control and the trabecular structure being broken. Magnification, x200. LM, light microscopy.

significant difference in IOP was identified between the 4th and 16th week after operation in the control group $(\mathrm{P}>0.05$; Fig. 8).

\section{Discussion}

Bone cement is widely used in hip replacements, but the potential clinical complications of its use have been largely unrecognized or ignored $(4,5)$. In the present study, a rabbit model of the PFMC was successfully established, the cavity blocked with bone cement and the BMD and IOP of the distal femur measured; structural changes in the articular cartilage and subchondral bone of the distal femur were also observed. The BMD of the distal femur decreased significantly at 4 weeks following bone cement treatment but was increased at week 16 following surgery, and this was significantly higher compared with the control group BMD. The IOP of the distal femur increased significantly after intervention and continued to be significantly higher compared with the control group. LM and TEM analyses showed that distal femur articular cartilage and subchondral bone were severely affected by the specific intervention, which suggested that the effect of bone cement on BMD, IOP, articular cartilage and subchondral bone of the distal femur should be considered when considering a hip replacement with bone cement fixation.

IOP is the pressure produced by the blood flow of the phalanx in the intramedullary cavity or the interosseous space and is the most reliable index to gauge intraosseous hemodynamics and the state of intraosseous circulation (20). Porsch et al (21) used modern bone cement implantation technology to set up pressure measuring devices in different positions of femur. They observed that bone cement and prosthesis implantation could lead to a significant increase in IOP (21). Welch et al (22) and Otto and Matis (23) also found that intramedullary filling with cement could lead to long-term high pressure in bone in animal experiments. These studies confirmed that the intramedullary pressure of the distal femur increased significantly after bone cement implantation, and it 
a

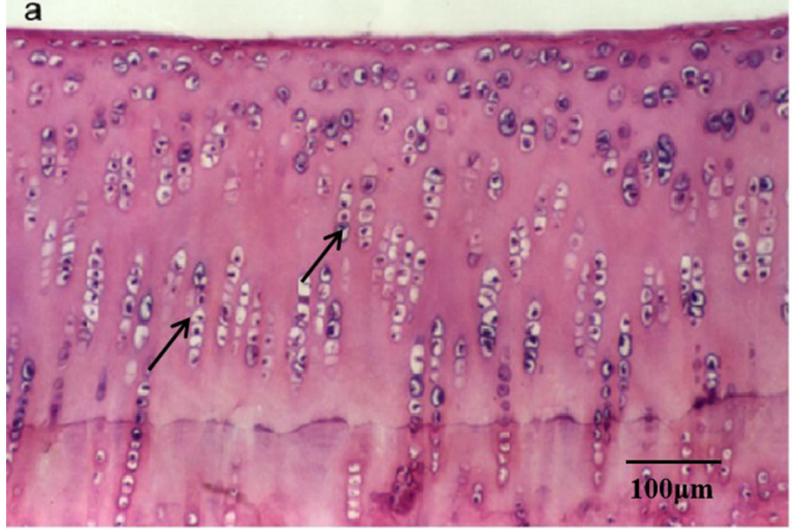

b

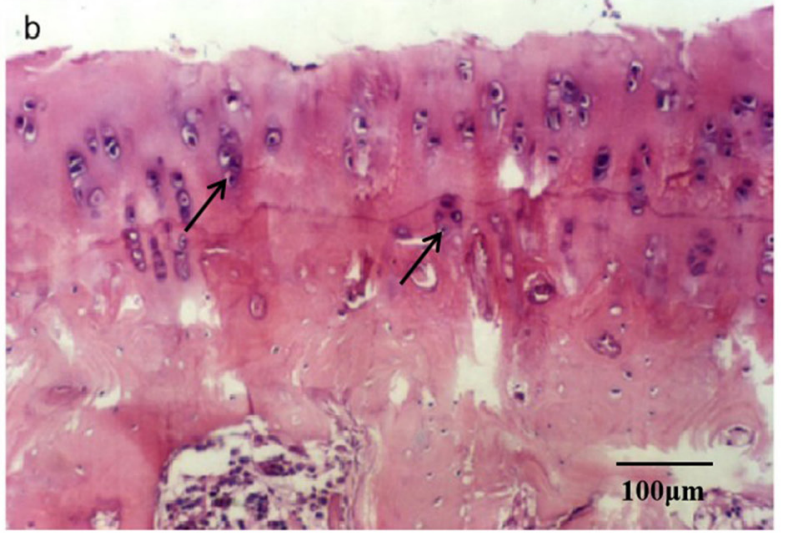

Figure 4. H\&E staining. (a) Articular cartilage in the control group under LM at the 16th week after intervention. The arrows indicate that the surface of cartilage is smooth and evenly distributed. (b) Articular cartilage of experimental group under LM at the 16th week after intervention. The arrows indicate that the cartilage layer became thin, the chondrocyte arrangement was disordered, and their quantity decreased. Magnification, x200. LM, light microscopy.

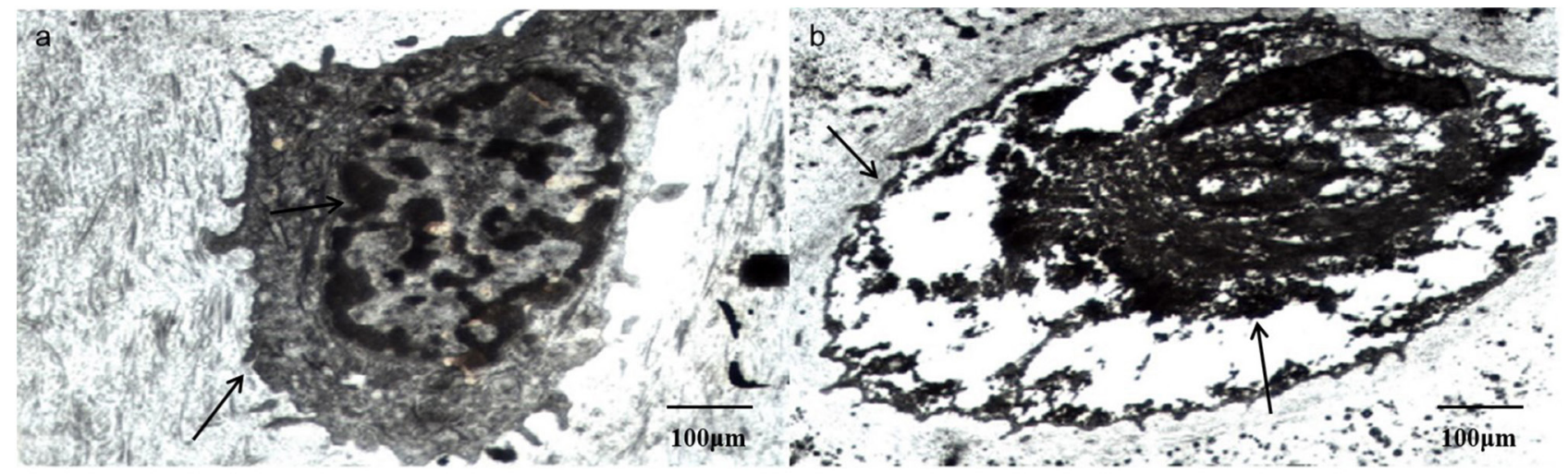

Figure 5. (a) Articular cartilage in the control group under TEM at the 16th week after intervention. The arrow indicates that the nucleus was large and complete and chromatin was abundant and evenly distributed throughout. (b) Articular cartilage of experimental group under TEM at the 16th week after intervention. The arrows indicate that the general structure of the chondrocytes was disorganized. Magnification, x12,000. TEM, transmission electron microscopy
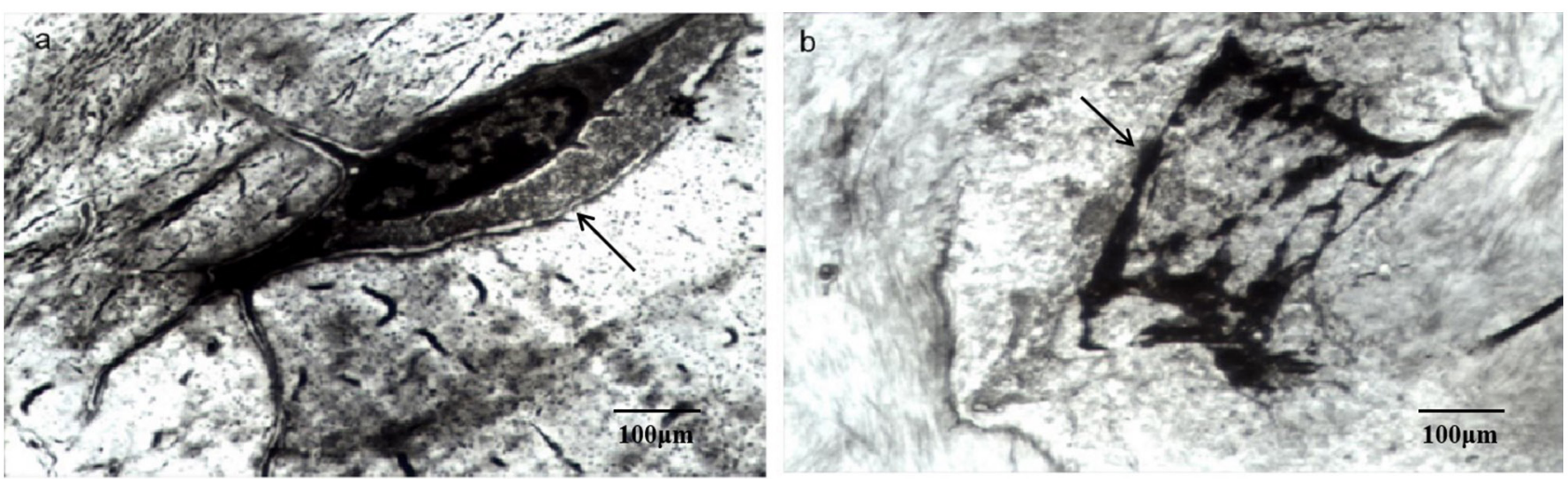

Figure 6. (a) Bone cells in the control group under TEM at the 16th week after intervention. The arrow indicates that osteocytes are rich in cytoplasm and endoplasmic reticulum and have a well-developed Golgi complex. (b) Bone cells on the experimental group under TEM at the 16th week after intervention. The arrow indicates that the continuity of cell membrane and nuclear membranes was interrupted, with a disappearance of cytoplasmic components. Magnification, x12,000. TEM, transmission electron microscopy.

continued to be significantly higher than that of the control group. This may be due to the long-term occlusion of the medullary cavity caused by the implantation of bone cement, which not only reduces the volume of the medullary cavity, but also destroys blood circulation in the bone and the pulp during the filling process of bone cement. This could further cause a series of hemodynamic changes resulting in increased IOP. The damage caused by bone cement implantation is a complicated one, and it likely takes a long time to repair. Therefore, intramedullary high pressure will exist for a long time after 


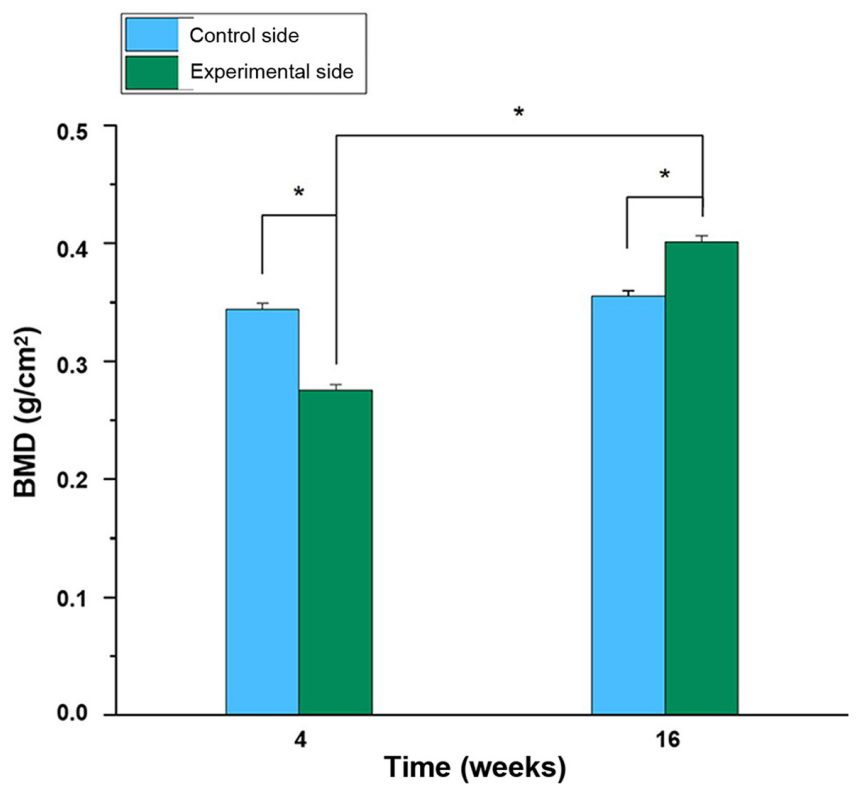

Figure 7. BMD in both groups at the 4 th and 16 th week after surgery. ${ }^{*} \mathrm{P}<0.05$. BMD, bone mineral density.

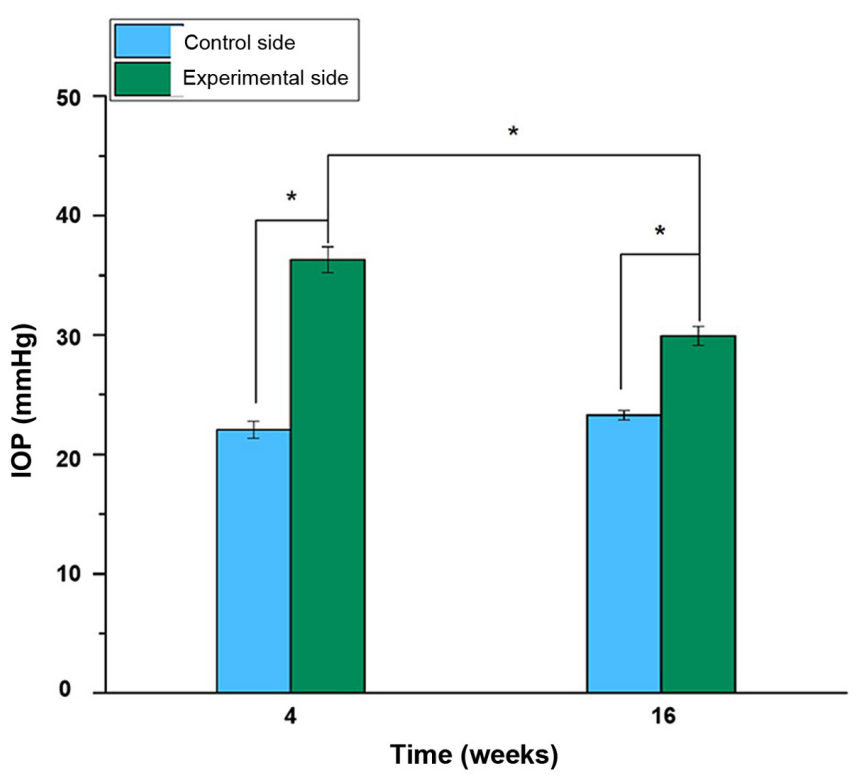

Figure 8 . IOP in both groups at the 4 th and 16 th week after surgery. ${ }^{*} \mathrm{P}<0.05$. IOP, intraosseous pressure.

bone cement implantation $(22,23)$. In turn, this increase in IOP could reduce blood flow in the bone marrow, which could lead to further dysfunction of the intraosseous vein reflux and a compression of the surrounding tissue, and this congestion may further reduce arterial blood flow, eventually causing irreversible subchondral ischemic necrosis (24).

The results of the present study indicated that bone cells in the subchondral bone of the proximal femur were affected by cement filling, including necrotic chondrocytes and disrupted bone microstructures. At the 16th week of bone cement occlusion, the cartilage layer of the distal femoral articular surface was thinner, the arrangement of chondrocytes was disordered and the number of cells significantly lowered. Although the underlying mechanism of this pathological change is still unknown, it can be hypothesized that tissue metabolic disorders owing to disrupted blood circulation might be the major cause. In addition, a number of other factors, such as necrosis of subchondral bone and accumulation of metabolites, could have aggravated the cartilage damage and resulted in degeneration of the distal joint $(25,26)$. A series of changes caused by toxicity, fever and osteolysis of bone cement may also accelerate the degeneration of the distal femoral articular surface. Further studies are needed to determine the precise contributions of these pathological processes to changes in IOP, BMD and bone and cartilage histology.

BMD refers to bone mineral content per unit bone tissue, which is a standard parameter to quantify bone quality (27). It can be used as a parameter to gauge the influence of cemented artificial joint implantation on the bone quality (28). For example, when van Loon et al (29) used dual energy X-ray absorptiometry to measure bone density in patients with a loosening prosthetic following artificial joint replacement, it was found that the loss of bone mass stimulated by bone cement was the main cause of the artificial joint loosening. Similarly, Kramhoft et al (30) also found that bone cement implantation reduced BMD and inhibited bone reconstruction and mineralization in dogs. The present results indicated that the BMD of the distal femur decreased significantly after bone cement blocked the PFMC, which may be due to the dual effects of high IOP and the actions of bone cement itself. It is well known that the implantation of bone cement into the medullary cavity leads to high IOP, which increases hypoxia at the distal end of the femur (31). This, in turn, could seriously affect bone and cartilage metabolism in the distal femur and result in a large loss of local bone mass (10).

In addition, particles from bone cement implantation can cause histochemical reactions, stimulating macrophages and giant cells to secrete a variety of factors related to bone resorption; therefore, bone cement might promote bone resorption in different ways (32). However, the results of the present study suggested that over time, BMD of the distal femur gradually increased and eventually exceeded normal BMD levels. This, at first glance, might be treated as good news, but further analysis found that this might not be a desirable outcome. In fact, this phenomenon may be due to abnormal bone repair and reconstruction, which can result in bone deposition and bone sclerosis (33). One previous study also reported an increase in BMD under pathological conditions (34).

The limitations of the present study include: i) Observation was for only 16 weeks, which may not be long enough to model artificial joint surgery; therefore, future study of the long-term effects of bone cement on the distal femur are warranted; ii) only a limited number of parameters were assessed in this study; therefore, more clinically relevant parameters are warranted for future studies; and iii) species-specific and model-specific features might argue against the clinical relevance of the present model to clinical hip replacements in humans. Future studies will test this hypothesis and other clinically associated or relevant models are warranted. Overall, the present data support the idea that cement treatment could potentially increase IOP, disrupt the BMD and perturb the local articular cartilage and subchondral bone structure. 
In summary, blockage of the PFMC with bone cement results in an increase in the IOP of the distal femur, changes in BMD and damage to the subchondral bone and articular cartilage. Further studies are warranted to further confirm this finding in the clinic and to investigate the mechanisms underlying how bone cement occlusion in the PFMC exactly affects the distal femur.

\section{Acknowledgements}

Not applicable.

\section{Funding}

This project was supported by The Self-financing Research Project of Guangxi Zhuang Autonomous Region Health Commission (grant no. Z20190244) and The National Natural Science Foundation of China (grant no. 81860274).

\section{Availability of data and materials}

All data generated and/or analyzed during the present study are included in this published article.

\section{Authors' contributions}

GPL and LCX contributed to the study design, manuscript preparation and data collection. GPL and LCX performed all the experiments. MZ performed the data analysis. JMZ contributed to the conception and design of the study. GPL and LCX confirmed the authenticity of all the raw data. All authors have read and approved the final manuscript.

\section{Ethics approval and consent to participate}

The Institutional Animal Care and Use Committee approved the use of rabbits in this study, and the study protocol was approved by The Ethics Review Board of Guangxi Medical University (Nanning, China). The study complied with the National Institutes of Health Guide for Care and Use of Laboratory Animals (Publication No. 85-23).

\section{Patient consent for publication}

Not applicable.

\section{Competing interests}

The authors declare that they have no competing interests.

\section{References}

1. Blankstein M, Lentine B and Nelms NJ: The use of cement in hip arthroplasty: A contemporary perspective. J Am Acad Orthop Surg 28: e586-e594, 2020.

2. Beckmann NA, Bitsch RG, Gondan M, Schonhoff M and Jaeger S: Comparison of the stability of three fixation techniques between porous metal acetabular components and augments Bone Joint Res 7: 282-288, 2018.

3. Amirouche F, Solitro G, Broviak S, Goldstein W, Gonzalez M and Barmada R: Primary cup stability in THA with augmentation of acetabular defect. A comparison of healthy and osteoporotic bone. Orthop Traumatol Surg Res 101: 667-673, 2015.
4. Kim SC, Ohashi H, Oonishi $\mathrm{H}$ and Oonishi H: Histologic findings at 14 and 18 years after cemented total hip arthroplasty with interface bioactive bone cement technique. J Arthroplasty 22: 1067-1069, 2007.

5. Singh V, Bhakta P, Zietak E and Hussain A: Bone cement implantation syndrome: A delayed postoperative presentation. J Clin Anesth 31: 274-277, 2016.

6. Vanderstappen J, Simon JP and Bellemans J: Radiographic analysis of a bone plug in 275 primary cemented total hip arthroplasties. Acta Orthop Belg 78: 350-356, 2012.

7. Motobe T, Hashiguchi T, Uchimura T, Yamakuchi M, Taniguchi N, Komiya S and Maruyama I: Endogenous cannabinoids are candidates for lipid mediators of bone cement implantation syndrome. Shock 21: 8-12, 2004.

8. Morda M, Pini S, Celli F, Casella F, Parchi P, Piolanti N, Marchetti S and Scaglione M: Bone cement implantation syndrome: A thromboelastographic study of the effect of bone cement on coagulation. J Biol Regul Homeost Agents 31: 121-127, 2017.

9. Yoon BH, Ha YC, Lee YK and Koo KH: Postoperative deep infection after cemented versus cementless total hip arthroplasty: A meta-analysis. J Arthroplasty 30: 1823-1827, 2015.

10. Xi LC, Li HY, Zhang M and Huang SC: Effects of bone cement filling in rabbit proximal femoral medullary cavity on distal femoral blood flow and metabolism. J Int Med Res 46: 5237-5244, 2018.

11. Robertson $\mathrm{C}$, Coutts $\mathrm{F}$ and Bell J: Investigation of anterior knee pain after total hip replacement: A pilot study. Physiother Res Int 12: 25-28, 2007.

12. Bartlett DH and Silk SB: Office of laboratory animal welfare comments. Zebrafish 13: 563-564, 2016.

13. Ni GX, Lu WW, Chiu KY, Li ZY, Fong DY and Luk KD: Strontium-containing hydroxyapatite (Sr-HA) bioactive cement for primary hip replacement: An in vivo study. J Biomed Mater Res B Appl Biomater 77: 409-415, 2006.

14. Miyanishi K, Yamamoto T, Irisa T, Yamashita A, Jingushi S, Noguchi Y and Iwamoto Y: Bone marrow fat cell enlargement and a rise in intraosseous pressure in steroid-treated rabbits with osteonecrosis. Bone 30: 185-190, 2002.

15. Beverly M and Murray D: Factors affecting intraosseous pressure measurement. J Orthop Surg Res 13: 187, 2018.

16. Pan S, Deng X, Sun S, Lai X, Sun L, Li Q, Xiang L, Zhang L and Huang Y: Black tea affects obesity by reducing nutrient intake and activating AMP-activated protein kinase in mice. Mol Biol Rep 45: 689-697, 2018.

17. Karawya S, Said DG, Salaheldin MM and Zaky I: Impact of intravitreal injection of bevacizumab (Avastin) on rabbit's choroid and retina. Middle East Afr J Ophthalmol 15: 67-72, 2008.

18. Khoo BC, Brown K, Cann C, Zhu K, Henzell S, Low V, Gustafsson S, Price RI and Prince RL: Comparison of QCT-derived and DXA-derived areal bone mineral density and T scores. Osteoporos Int 20: 1539-1545, 2009.

19. Pennypacker BL, Oballa RM, Levesque S, Kimmel DB and Duong LT: Cathepsin K inhibitors increase distal femoral bone mineral density in rapidly growing rabbits. BMC Musculoskelet Disord 14: 344, 2013.

20. Beverly M, Mellon S, Kennedy JA and Murray DW: Intraosseous pressure during loading and with vascular occlusion in an animal model. Bone Joint Res 7: 511-516, 2018.

21. Porsch M, Schmidt J, Brimmers P, Menne A and Merkle W: Intrafemoral pressure measurement in different cement removal procedures during hip prosthesis replacement operations-experimental study with cadaver femora. Biomed Tech (Berl) 43: 53-57, 1998 (In German).

22. Welch RD, Johnston CE II, Waldron MJ and Poteet B: Bone changes associated with intraosseous hypertension in the caprine tibia. J Bone Joint Surg Am 75: 53-60, 1993.

23. Otto $\mathrm{K}$ and Matis U: Changes in cardiopulmonary variables and platelet count during anesthesia for total hip replacement in dogs. Vet Surg 23: 266-273, 1994.

24. Blankstein M, Widmer D, Gotzen M, Hofmann-Fliri L, Richards RG, Gueorguiev B and Windolf M: Assessment of intraosseous femoral head pressures during cement augmentation of the perforated proximal femur nail antirotation blade. J Orthop Trauma 28: 398-402, 2014.

25. Li C, Kotha S and Mason J: Evaluation of the effects of implant materials and designs on thermal necrosis of bone in cemented hip arthroplasty. Biomed Mater Eng 13: 419-428, 2003. 
26. Radin EL and Rose RM: Role of subchondral bone in the initiation and progression of cartilage damage. Clin Orthop Relat Res: 34-40, 1986.

27. Fonseca H, Moreira-Gonçalves D, Coriolano HJ and Duarte JA: Bone quality: The determinants of bone strength and fragility. Sports Med 44: 37-53, 2014.

28. Peitgen DS, Innmann MM, Merle C, Gotterbarm T, Moradi B and Streit MR: Periprosthetic bone mineral density around uncemented titanium stems in the second and third decade after total hip arthroplasty: A DXA study after 12, 17 and 21 years. Calcif Tissue Int 103: 372-379, 2018.

29. van Loon CJ, de Waal Malefijt MC, Buma P, Verdonschot $\mathrm{N}$ and Veth RP: Femoral bone loss in total knee arthroplasty. A review. Acta Orthop Belg 65: 154-163, 1999.

30. Kramhoft M, Bodtker S, Nimb L and Jensen JS: Variation of cortical hypertrophy depending on the medullary filling material. An experimental study of canine tibial diaphysis. J Arthroplasty 8: 555-560, 1993.
31. Diehl K: Bone stress and reconstruction of the bone at the alloarthroplasty of the upper end of the femur with cement bonding (author's transl). Arch Orthop Unfallchir 83: 9-28, 1975 (In German).

32. Nakashima Y, Sun DH, Trindade MC, Chun LE, Song Y, Goodman SB, Schurman DJ, Maloney WJ and Smith RL: Induction of macrophage $\mathrm{C}-\mathrm{C}$ chemokine expression by titanium alloy and bone cement particles. J Bone Joint Surg Br 81: 155-162, 1999.

33. Karsdal MA, Bay-Jensen AC, Lories RJ, Abramson S, Spector T, Pastoureau P, Christiansen C, Attur M, Henriksen K, Goldring SR and Kraus V: The coupling of bone and cartilage turnover in osteoarthritis: Opportunities for bone antiresorptives and anabolics as potential treatments? Ann Rheum Dis 73: 336-348, 2014.

34. Lajeunesse D and Reboul P: Subchondral bone in osteoarthritis: A biologic link with articular cartilage leading to abnormal remodeling. Curr Opin Rheumatol 15: 628-633, 2003. 